\title{
Matrices de transición, una herramienta para eva- luar la sostenibilidad de sistemas agroforestales
}

\author{
Iransition Matrix, a tool to assess the sustainability of agroforestry systems
}

Wilson Antonio Calero Borge ${ }^{1}$

\section{Resumen}

$\mathrm{F}^{1}$ trabajo se desarrolló en la Reserva Indígena de Talamanca, Costa Rica. Se midieron 64 parcelas de Eacao (C) y banano (B), para determinar la sostenibilidad del aprovechamiento de madera que realizan los agricultores indígenas, midiendo el incremento de madera de laurel (Cordia alliodora) y cedro amargo (Cedrela odorata L.) de regeneración natural en estos sistemas agroforestales a través de matrices de transición como herramienta que permite calcular las tasas de paso o permanencia en diferentes clases diamétricas de individuos en crecimiento. Se realizó inventario de laurel y cedro amargo y se midieron árboles con $\mathrm{d} \geq 4 \mathrm{~cm}$. El volumen comercial $(\mathrm{Vc})(\mathrm{d} \geq 45 \mathrm{~cm})$ se proyectó con matrices de transición tipo Usher. Se encontró que el aprovechamiento de madera en los SAF evaluados es sostenible, puesto que hay incrementos de madera y de densidad de individuos en los cacaotales, esto indica el buen manejo que realizan los productores indígenas en los sistemas agroforestales. Se recomienda el uso de las matrices de transición como herramienta para evaluar la sostenibilidad de estos sistemas productivos.

Palabras clave: sistemas agroforestales; regeneración natural; matrices de transición; sostenibilidad.

\section{Summary}

This work was developed in the Indigenous Reserve of Talamanca, Costa Rica. 64 plots of cocoa (C) and banana (B) were measured to determine the sustainability of the use of wood by indigenous farmers, measuring the increase in wood of laurel (Cordia alliodora) and cedro amargo (Cedrela odorata L.) natural regeneration in these agroforestry systems through transition matrix as a tool that allows to calculate the rates of passage or permanence in different diametric classes of individuals growing. An inventory of laurel and cedro amargo was carried out and trees with $\mathrm{d}>4 \mathrm{~cm}$ were measured. The commercial volume $(\mathrm{Vc})(\mathrm{d}>45 \mathrm{~cm})$ was projected with Usher-type transition matrix. It was found that the use of wood in the evaluated AFS is sustainable, since there are increases in wood and density of individuals in the cacao plantations, this indicates the good management carried out by indigenous producers in agroforestry systems. The use of transition matrix is recommended as a tool to evaluate the sustainability of these productive systems.

Key Words: agroforestry systems; natural regeneration; transition matrix, sustainability

\section{Introducción}

Los Sistemas Agroforestales (SAF) son formas de uso de la tierra que se caracterizan por contener en sus diversos componentes grandes cantidades de biomasa y abundantes acumulaciones de elementos como el carbono (Nair 1997). Así mismo, el componente arbóreo maderable presente en los SAF es un

\footnotetext{
Master en Agroforestería Tropical, Coordinador de Investigación y Postgrado de la Universidad de las Regiones Autónomas de la Costa Caribe Nicaragüense-Recinto Universitario Nueva Guinea. Correo: investigación.ng@uraccan.edu.ni ORCID: https://orcid.org/0000-0003-4243-0490
}

Recibido: 10/11/2017 Aprobado: 23/11/2017 
recurso valioso que brinda diferentes beneficios al agricultor, presta servicios ambientales como captura de carbono, protección del suelo, conservación de biodiversidad, entre otros (Barrance et al, 2003; Albertin y Nair, 2004).

El cacao y banano son cultivos que las comunidades indígenas, en este caso Talamanca en Costa Rica, han cultivado de antaño, bajo diferentes doseles de sombra y constituyen sus principales actividades económicas. El laurel (Cordia alliodora) y cedro amargo (Cedrela odorata) son las principales especies maderables en las fincas de indígenas de Talamanca (Suatunce, 2002). Estas especies son muy apreciadas por su madera, abundante regeneración natural, rápido crecimiento, sombra rala, y autopoda, en el caso del laurel. Los agricultores obtienen ingresos económicos al aprovecharlas en épocas de baja producción o bajos precios del cacao (Albertin y Nair, 2004; Russell y Francell, 2004).

Considerando la diversidad de especies en los SAF, el aprovechamiento de madera que realiza el productor indígena, el reclutamiento y la muerte de individuos, el presente trabajo propone el uso de matrices de transición como una herramienta para estimar la sostenibilidad de los SAF de indígenas de Talamanca.

\section{Revisión de literatura}

\section{Regeneración y crecimiento del laurel en SAF}

El éxito del establecimiento de la regeneración natural de laurel depende de la protección contra animales que depredan las semillas, en potreros, la regeneración natural está muy influenciada por los factores edáficos en sus primeras etapas y por manejo y uso anterior de la tierra en las sucesivas etapas de desarrollo de la planta (Camargo et al. 2000).

\section{Modelos de crecimiento}

Un modelo es una abstracción o una representación simplificada de algunos aspectos de la dinámica natural de un rodal y puede involucrar crecimiento, mortalidad y otros cambios en la estructura y composición. Un modelo puede predecir el crecimiento y rendimiento del rodal bajo diversas condiciones y se pueden agrupar asi; a nivel de rodal, de árboles individuales y por clase de tamaños (Vanclay, 1994). Existen tres variaciones de estas aproximaciones: las cadenas de Markov, las matrices de Usher y sus generalizaciones (Vanclay,1994; Moscovich, 2004).

\section{Las cadenas de Markov}

En las cadenas de Markov, un árbol en una clase diamétrica, durante el próximo período puede permanecer en la clase, crecer y pasar a la siguiente clase, ser cosechado o morir. Las probabilidades de paso son expresadas como una matriz y son usadas para predecir cambios en un intervalo de tiempo o sobre varios intervalos de tiempo $h=M_{t} * V_{0}$; donde $M_{t}$ es la matriz que contiene las probabilidad de movimiento y $V_{0}$ y $V_{n}$ son los vectores que describen los estados inicial y final, respectivamente (Vanclay, 1994).

\section{Las matrices de Usher}

Las matrices de Usher intentan reducir el largo número de parámetros requeridos para formar una cadena de Markov, seleccionando el intervalo de tiempo y una amplitud de clase en la que un árbol durante ese intervalo no pueda crecer más que una clase, permite una sustancial reducción en el número de parámetros a ser estimados, entonces, la matriz puede ser reducida a cuatro vectores: crecimiento, fecundidad, cosecha y mortalidad (Vanclay, 1994).

\section{Aplicaciones de los modelos matriciales de Usher}

Diversas aplicaciones de estos modelos matriciales para árboles en sistemas agroforestales han sido desarrolladas. Somarriba (2001) modeló la dinámica poblacional de Acacia pennatula. La ecuación empleada fue $n_{t+1}=M * n_{t}+z$, en donde $n_{t}$ y $n_{t+1}$, denota los vectores de las distribuciones diamétricas en el periodo $t$ y $t+1$, y $z$ es el vector de reclutamiento. Los coeficientes de transición de la matriz $M, e_{i j}$ y $r_{i j}$ fueron calculados con base a la tasa anual de paso y tasa anual de permanencia, respectivamente, y ajustados por un factor de supervivencia por clase diamétrica.

En la zona de Talamanca, Costa Rica, en sistemas productivos de cacao y banano, para la especie Cordia alliodora en condiciones de regeneración natural, Suárez (2001) desarrolló un modelo de matrices de transición modificada (Somarriba, 2001) 
del tipo $n_{t+1}=M^{*} n_{t}$, donde los vectores $n_{t}$ y $n_{t+1}$, denotan la distribución diamétrica entre los años sucesivos $t$ y $t+1$ y $M$ es la matriz de los coeficientes de transición y de permanencia (Suárez, 2001).

\section{Materiales y métodos}

\section{Población de estudio}

La población de estudio consistió en los cacaotales y bananales de indígenas Bribri y Cabécar, en Talamanca, Costa Rica, para ello se midieron 64 parcelas que han sido medidas cada 5 años.

\section{Modelación de la dinámica poblacional de laurel en sistemas agroforestales}

Se proyectó la dinámica poblacional desarrollando un modelo de matrices de transición tipo Usher que proponen Somarriba (2001) y Suárez (2001). La población fue dividida en clases diamétricas de $5 \mathrm{~cm}$. El diámetro mínimo de corta fue establecido en $45 \mathrm{~cm}$.

La iteración se realizó por periodos de un año hasta una proyección de cinco años para determinar la capacidad de producción de madera y comportamiento de la población de laurel de los SAF en estudio. La ecuación matricial empleada fue la siguiente:

$$
n_{t+1}=M * n_{t}+R
$$

Donde los vectores $n_{t+1}$ y $n_{t}$ denotan la distribución diamétrica (árboles ha ${ }^{-1}$ ) entre los años sucesivos $t$ y $t+1, M$ es la matriz de coeficientes de transición y $R$ es el reclutamiento de individuos en el periodo que se analiza. La ecuación $n_{t+1}=M * n_{t}+R$ devuelve en cada iteración en la clase diamétrica superior la densidad de árboles que alcanzan el diámetro mínimo de corta.

Suponiendo una población de $z$ clases diamétricas $\left(z_{1}, z_{2 \ldots} z l\right)$ en el año, en donde $z l$ es la clase diamétrica de los árboles de cosecha, la proyección de la población en el año $t+1$ con la ecuación $n_{t+1}=M * n_{t}+R$ en forma matricial es:

$$
\left|\begin{array}{c}
a \\
z_{1, t+1} \\
z_{2, t+1} \\
\cdot \\
z_{l, t+1}
\end{array}\right|_{=}\left|\begin{array}{cccccc}
1 & 0 & 0 & 0 & 0 & 0 \\
e_{j} & r_{i} & 0 & 0 & 0 & 0 \\
0 & e_{j} & r_{i} & 0 & 0 & 0 \\
0 & 0 & . & . & 0 & 0 \\
0 & 0 & 0 & . & \cdot & 0 \\
0 & 0 & 0 & 0 & e_{j} & r_{i}
\end{array}\right|_{*}\left|\begin{array}{c}
a \\
z_{1, t} \\
z_{2, t} \\
- \\
z_{l, t}
\end{array}\right|+\mathrm{R}
$$

Dónde:

- $e_{\ddot{j}}$ : Coeficientes de transición del periodo $i$ al periodo $j$

$e_{i j}=p_{i j} \cdot S_{i}$

- $\boldsymbol{r}_{i \boldsymbol{i}}$ : Coeficientes de permanencia en el periodo $i$

$r_{i i}=q_{i i} \cdot S_{i}$ 
El cálcculo de estos coeficientes fue derivado de la estimación de una tasa anual de paso $\left(p_{i j}\right)$ de clase $i$ a clase $j$ y una tasa anual de permanencia $\left(q_{i i}\right)$ en la misma clase $i$ ajustados por un factor de supervivencia anual por clase $(\mathrm{Si})$, entonces:

$$
p_{\ddot{y}}=\frac{i_{d}}{K_{i}}
$$

Donde $i_{d}$ denota el incremento corriente anual en diámetro en la clase $i$ estimado con la primera derivada de la ecuación de crecimiento de Chapman Richards (Suárez, 2001) y $K_{i}$ el intervalo de clase $(5 \mathrm{~cm}$ ).

$$
i_{d}=a * b * c * e^{-b t}\left(1-e^{-b t}\right)^{c-1}
$$

Dónde:

$a ; b ; c$ : Parámetros de la ecuación de regresión

$e:$ Base de los logaritmos

$t$ : Edad para alcanzar la categoría diamétrica

- Entonces la tasa anual de permanencia se calculó de la siguiente manera:

$$
q_{i i}=1-P_{i j}
$$

Dónde:

$\boldsymbol{q}_{\ddot{\boldsymbol{u}}}:$ Tasa anual de permanencia

$p_{\ddot{j}}$ : Tasa anual de paso de la clase $i$ a la clase $j$

Cálculo de la supervivencia

La supervivencia anual por clase () se estimó con base en la distribución diamétrica y las tasas de aprovechamiento anual por clase diamétrica para laurel. La ecuación que se empleó fue la siguiente:

$$
S_{i}=\frac{\left(N_{i}-T_{i}\right)}{N_{i}}
$$

Dónde:

$S_{i}$ : Es la supervivencia en la clase $\boldsymbol{i}$

$N_{i}$ : es el número de individuos en la clase diamétrica $\boldsymbol{i}$

$T_{i}$ : es la tasa de aprovechamiento anual para la clase diamétrica $\boldsymbol{i}$.

\section{Cálculo del reclutamiento anual}

Se estimó comparando las curvas de distribución diamétrica de los inventarios sucesivos. La diferencia entre ambas curvas permitió obtener el número de individuos que se incorporaron a las clases diamétricas en el periodo, la ecuación fue la siguiente: 
Dónde:

$R_{i}$ : Tasa anual de reclutamiento (árboles $\mathrm{ha}^{-1} \mathrm{año}^{-1}$ )

$N_{\text {iactual }}:$ Individuos en la clase diamétrica $i$ por hectárea en el inventario actual.

$$
R_{i}=\frac{\left(N_{\text {iactual }}-N_{\text {ibase }}\right)}{5}
$$

$N_{\text {ibase }}$ : Individuos en la clase diamétrica $i$ por hectárea en el inventario anterior.

Cálculo de la tasa de aprovechamiento de laurel

El volumen de madera aprovechado anualmente en las parcelas se estimó mediante recorridos por la parcela, el propietario indicó los tocones aprovechados y el año de extracción. Se midió la altura de corte y el diámetro a todos los tocones aprovechados en el periodo. El diámetro se midió en dos direcciones tratando de encontrar los puntos intermedios del tocón debido a la presencia de aletones. Posteriormente, el volumen se estimó empleando la ecuación de Somarriba y Beer (1987):

$$
V=e^{-9,62+2,697 \ln }
$$

Donde:

$V$ : Volumen del fuste con corteza $\left(\mathrm{m}^{3}\right)$

$e:$ Base de los logaritmos naturales

$d$ : Diámetro a la altura del pecho $(\mathrm{cm})$

Debido a que las alturas de corte son variables, se estandarizó el diámetro de los tocones a una altura de corte de 1,3 m (dap) con la ecuación de Pérez (1954) y Suárez (2001):

$$
d=-136,90622+37,51902 \ln (d t)+8,15199 \ln (h t)
$$

Donde:

$d t$ : Diámetro a la altura del tocón (cm)

ht: Altura del tocón (cm)

d: Diámetro del tocón a 1,30 m de altura (cm)

\section{Análisis de escenarios}

El análisis de la dinámica poblacional de laurel se realizó a nivel de poblaciones y de parcelas, para evaluar el efecto como resultado de cambios en las prácticas de manejo, se simuló para cinco años el comportamiento con diferentes tasas de sobrevivencia y reclutamiento. Los escenarios fueron: 1) Proyección 
a cinco años con los datos observados (árboles ha ${ }^{-1}$ ), 2) 100\% de sobrevivencia en las clases diamétricas inferiores (dap < $45 \mathrm{~cm}$ ) y 3 ) Incrementos del 50\% en las tasas de reclutamiento.

\section{Proyección del rendimiento de madera}

Se realizó a partir de la distribución poblacional en clases diamétricas, con la siguiente ecuación se estimó la producción de madera $\left(\mathrm{m}^{3} \mathrm{ha}^{-1}\right.$ año $\left.\mathrm{o}^{-1}\right)$ para cada sistema agroforestal.

$$
I C A V c=V c_{i+1}-V c_{i}
$$

Donde:

$I C A V C:$ Incremento corriente anual del volumen comercial $\left(\mathrm{m}^{3} \mathrm{ha}^{-1} \mathrm{año}^{-1}\right)$

$V c_{i+1}$ : Volumen comercial proyectado en el año siguiente $\left(\mathrm{m}^{3} \mathrm{ha}^{-1}\right.$ año $\left.{ }^{-1}\right)$

$V c_{i}$ : Volumen comercial proyectado en el año actual $\left(\mathrm{m}^{3} \mathrm{ha}^{-1} \mathrm{año}^{-1}\right)$

Evaluación de la sostenibilidad del sistema agroforestal

Se asumió lo siguiente: si el incremento corriente anual del volumen comercial (ICAVc) es mayor que cero el aprovechamiento es sostenible, si es igual a cero está en equilibrio con el crecimiento de la población, si el $I C A V c$ es menor que cero el aprovechamiento es no sostenible. También se determinó el incremento periódico anual del volumen comercial $I P A V C$. La ecuación fue:

$$
I P A V c=\frac{\left(V c_{f}-V c_{i}\right)}{5}
$$

Dónde:

$I P A V C$ : Incremento periódico anual del volumen comercial proyectado $\left(\mathrm{m}^{3} \mathrm{ha}^{-1} \mathrm{año}^{-1}\right)$.

$V c_{f:}$ Volumen comercial $\left(\mathrm{m}^{3} \mathrm{ha}^{-1}\right)$ al final del periodo proyectado de cinco años.

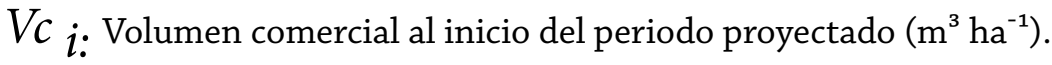

El análisis a nivel de parcelas se realizó calculando las tasas de sobrevivencia y reclutamiento anual, la distribución diamétrica fue la encontrada en cada parcela en este estudio.

Se realizaron iteraciones para cinco años y se encontraron los incrementos en densidad, si el incremento fue positivo se consideró que hay crecimiento de la población, si es negativo entonces hay disminución de la población, se encontraron las tasas anuales de incremento considerando el período de proyección, las ecuaciones fueron las siguientes:

$$
\Delta N=N_{\text {proy }}-N_{\text {base }}
$$

Dónde:

$\Delta N$ : Cambio en la densidad de árboles en inventarios sucesivos (árboles ha ${ }^{-1}$ ).

$N_{\text {proy }}$ : Densidad de árboles a cinco años de proyección (árboles ha ${ }^{-1}$ ) 
$N_{\text {base }}$ : Inventario base en la parcela (ár $I P A N=\frac{\Delta N}{5 \text { años }}$

Dónde:

$I P A N$ : Incrementos periódicos anuales de la densidad (árboles $\mathrm{ha}^{-1} \mathrm{año}^{-1}$ )

$\Delta N$ : Cambios en la densidad de árboles en el periodo analizado (árboles ha ${ }^{-1}$ )

\section{Resultados y discusión}

\section{Aprovechamiento de madera de laurel en sistemas agroforestales de Talamanca}

Las tasas anuales de corta de laurel (Cuadro 1) son mayores en los cacaotales; en los bananales se extrae el 38\% de madera en relación a los cacaotales. En las laderas se extrae menos madera que en los valles (26\% con relación al valle). El volumen comercial representó el $84 \%$ del volumen total anual extraído en los valles y el $80 \%$ en las laderas.

Se están aprovechando $0,92 \mathrm{~m}^{3} \mathrm{ha}^{-1}$ año ${ }^{-1}$ lo que equivale a una extracción de $216 \mathrm{~m}^{3}$ en cinco años en 47 hectáreas, las tasas de extracción en los SAF de cacao y banano son de $1,16 \mathrm{~m}^{3} \mathrm{ha}^{-1}$ año ${ }^{-1}$ y o,67 $\mathrm{m}^{3} \mathrm{ha}^{-1}$ año ${ }^{-1}$ respectivamente, son superiores a las reportadas por Suárez (2001) para SAF de cacao y banano siendo de 0,89 y $0,11 \mathrm{~m}^{3} \mathrm{ha}^{-1}$ año ${ }^{-1}$ respectivamente.

Cuadro 1. Tasas anuales de corta de madera de laurel $\left(\mathrm{m}^{3} \mathrm{ha}^{-1}\right.$ año $\left.{ }^{-1}\right)$ en cacaotales y bananales en Talamanca, Costa Rica.

\begin{tabular}{|c|c|c|c|c|}
\hline \multirow{3}{*}{$\begin{array}{c}\text { Clase diamétrica } \\
(\mathrm{cm})\end{array}$} & \multicolumn{2}{|c|}{ Cacao } & \multicolumn{2}{|c|}{ Banano } \\
\hline & Valle & Ladera & Valle & Ladera \\
\hline & $\mathrm{m}^{3} \mathrm{ha}^{-1} \mathrm{año}^{-1}$ & $\mathrm{~m}^{3} \mathrm{ha}^{-1} \mathrm{año}^{-1}$ & $\left.\mathrm{~m}^{3} \mathrm{ha}^{-1} \mathrm{año}^{-1}\right)$ & $\mathrm{m}^{3} \mathrm{ha}^{-1} \mathrm{año} 0^{-1}$ \\
\hline $4-10,9$ & 0,00 & 0,00 & 0,00 & 0,00 \\
\hline $11-15,9$ & 0,00 & 0,00 & 0,02 & 0,00 \\
\hline $16-20,9$ & 0,01 & 0,04 & 0,02 & 0,00 \\
\hline $21-25,9$ & 0,00 & 0,01 & 0,00 & 0,00 \\
\hline $26-30,9$ & 0,02 & 0,01 & 0,02 & 0,00 \\
\hline $31-35,9$ & 0,00 & 0,00 & 0,00 & 0,00 \\
\hline $36-40,9$ & 0,18 & 0,02 & 0,03 & 0,12 \\
\hline $41-45,9$ & 0,07 & 0,07 & 0,05 & 0,00 \\
\hline $46-50,9$ & 0,20 & 0,01 & 0,09 & 0,00 \\
\hline $51-55,9$ & 0,33 & 0,00 & 0,05 & 0,25 \\
\hline $56-60,9$ & 0,18 & 0,00 & 0,00 & 0,61 \\
\hline $61-65,9$ & 0,34 & 0,00 & 0,09 & 0,00 \\
\hline $66-70,9$ & 0,32 & 0,00 & 0,00 & 0,00 \\
\hline $71-75,9$ & 0,14 & 0,00 & 0,00 & 0,00 \\
\hline $76-80,9$ & 0,21 & 0,00 & 0,00 & 0,00 \\
\hline $81-85,9$ & 0,00 & 0,00 & 0,00 & 0,00 \\
\hline$>86$ & 0,17 & 0,00 & 0,00 & 0,00 \\
\hline Volumen total & 2,17 & 0,16 & 0,37 & 0,98 \\
\hline V comercial ( $\geq 45 \mathrm{~cm}$ de dap) & 1,89 & 0,01 & 0,23 & 0,86 \\
\hline
\end{tabular}


Hay mayor extracción de árboles en los cacaotales, las mayores frecuencias de extracción correspondieron a individuos con dap $\geq 35 \mathrm{~cm}$ en cacaotales y bananales. La extracción de individuos jóvenes (dap $\leq 15$ $\mathrm{cm}$ ) es alta y similar en ambos sistemas agroforestales (Figura 1).

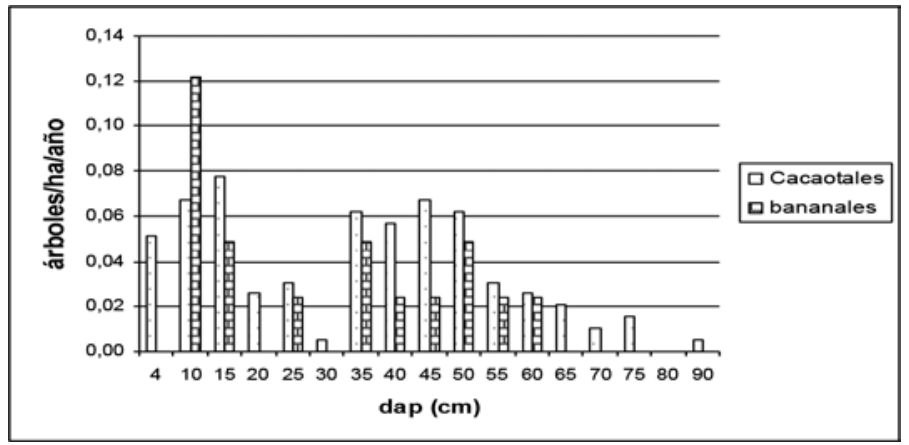

Figura 1. Diámetros de árboles de laurel aprovechados anualmente en cacaotales y bananales de Talamanca, Costa Rica.

\section{Proyección de la densidad, rendimiento e incremento del volumen comercial de madera de laurel en los sistemas agroforestales}

Las probabilidades de paso son muy semejantes para valle o ladera, ya sea cacao o banano (Cuadro 2). Las tasas de aprovechamiento se consideraron como las únicas fuentes de disminución de la población de laurel y fueron de 0,52 y o,26 árboles $\mathrm{ha}^{-1}$ año $^{-1}$ y el reclutamiento fue de 3,26 y 1,80 árboles ha ${ }^{-1}$ año ${ }^{-1}$ para bananales y cacaotales respectivamente, en general los bananales tienen los mejores resultados en estas dos variables, presentan las menores tasas de aprovechamiento y las mayores tasas de reclutamiento.

Cuadro 2. Probabilidades de paso ( $\left.{ }^{e_{i j}}\right)$ y de permanencia ( ${ }^{r_{i i}}$ ) por categorías diamétricas para las poblaciones de laurel.

\begin{tabular}{|c|c|c|c|c|c|c|c|c|c|}
\hline \multirow{3}{*}{$\begin{array}{c}\text { Clase diámetrica } \\
(\mathrm{cm})\end{array}$} & & \multicolumn{4}{|c|}{ Cacao } & \multicolumn{4}{|c|}{ Banano } \\
\hline & \multicolumn{2}{|c|}{ Valle } & \multicolumn{2}{|c|}{ Ladera } & \multicolumn{2}{|c|}{ Valle } & \multicolumn{2}{|c|}{ Ladera } & \multirow[b]{2}{*}{$r_{i i}$} \\
\hline & $\mathbf{j}$ & $e_{\ddot{j}}$ & $r_{i i}$ & $e_{i j}$ & $r_{i i}$ & $e_{i j}$ & $r_{i i}$ & $e_{\ddot{y}}$ & \\
\hline 4 & 10 & 0,54 & 0,37 & 0,39 & 0,52 & 0,49 & 0,50 & 0,42 & 0,57 \\
\hline 10 & 15 & 0,55 & 0,41 & 0,40 & 0,57 & 0,54 & 0,40 & 0,39 & 0,55 \\
\hline 15 & 20 & 0,53 & 0,44 & 0,38 & 0,60 & 0,54 & 0,45 & 0,38 & 0,61 \\
\hline 20 & 25 & 0,50 & 0,47 & 0,35 & 0,63 & 0,51 & 0,48 & 0,35 & 0,64 \\
\hline 25 & 30 & 0,47 & 0,50 & 0,32 & 0,65 & 0,47 & 0,50 & 0,32 & 0,66 \\
\hline 30 & 35 & 0,44 & 0,54 & 0,29 & 0,70 & 0,44 & 0,55 & 0,29 & 0,70 \\
\hline 35 & 40 & 0,39 & 0,55 & 0,24 & 0,70 & 0,38 & 0,55 & 0,24 & 0,69 \\
\hline 40 & 45 & 0,35 & 0,58 & 0,21 & 0,72 & 0,35 & 0,59 & 0,21 & 0,74 \\
\hline 45 & 50 & 0,30 & 0,60 & 0,17 & 0,73 & 0,31 & 0,62 & 0,17 & 0,76 \\
\hline 50 & 55 & 0,25 & 0,60 & 0,13 & 0,72 & 0,27 & 0,64 & 0,14 & 0,78 \\
\hline 55 & 60 & 0,22 & 0,65 & 0,10 & 0,77 & 0,22 & 0,66 & 0,10 & 0,78 \\
\hline 60 & 65 & 0,19 & 0,68 & 0,06 & 0,80 & 0,18 & 0,67 & 0,06 & 0,78 \\
\hline 65 & 70 & 0,13 & 0,61 & 0,03 & 0,71 & 0,17 & 0,82 & 0,04 & 0,95 \\
\hline 70 & 75 & 0,11 & 0,74 & 0,02 & 0,86 & 0,13 & 0,86 & 0,01 & 0,99 \\
\hline 75 & 80 & 0,07 & 0,72 & & & 0,09 & 0,90 & & \\
\hline 80 & 85 & 0,05 & 0,94 & & & 0,05 & 0,94 & & \\
\hline
\end{tabular}


$e_{i j}$ : coeficientes de paso; ${ }^{r_{i i}}$ : coeficientes de permanencia; i: clase diamétrica de permanencia; j: clase diamétrica de paso

En todas las poblaciones la curva se desplazará hacia la derecha haciendo moda en diámetros de 30 a $35 \mathrm{~cm}$ (Figura 2), lo que indica que no se producirán incrementos considerables, esto puede provocar una distribución poblacional donde las clases diamétricas inferiores tengan muy pocos individuos para mantener una adecuada estructura de individuos jóvenes y diámetros aprovechables.
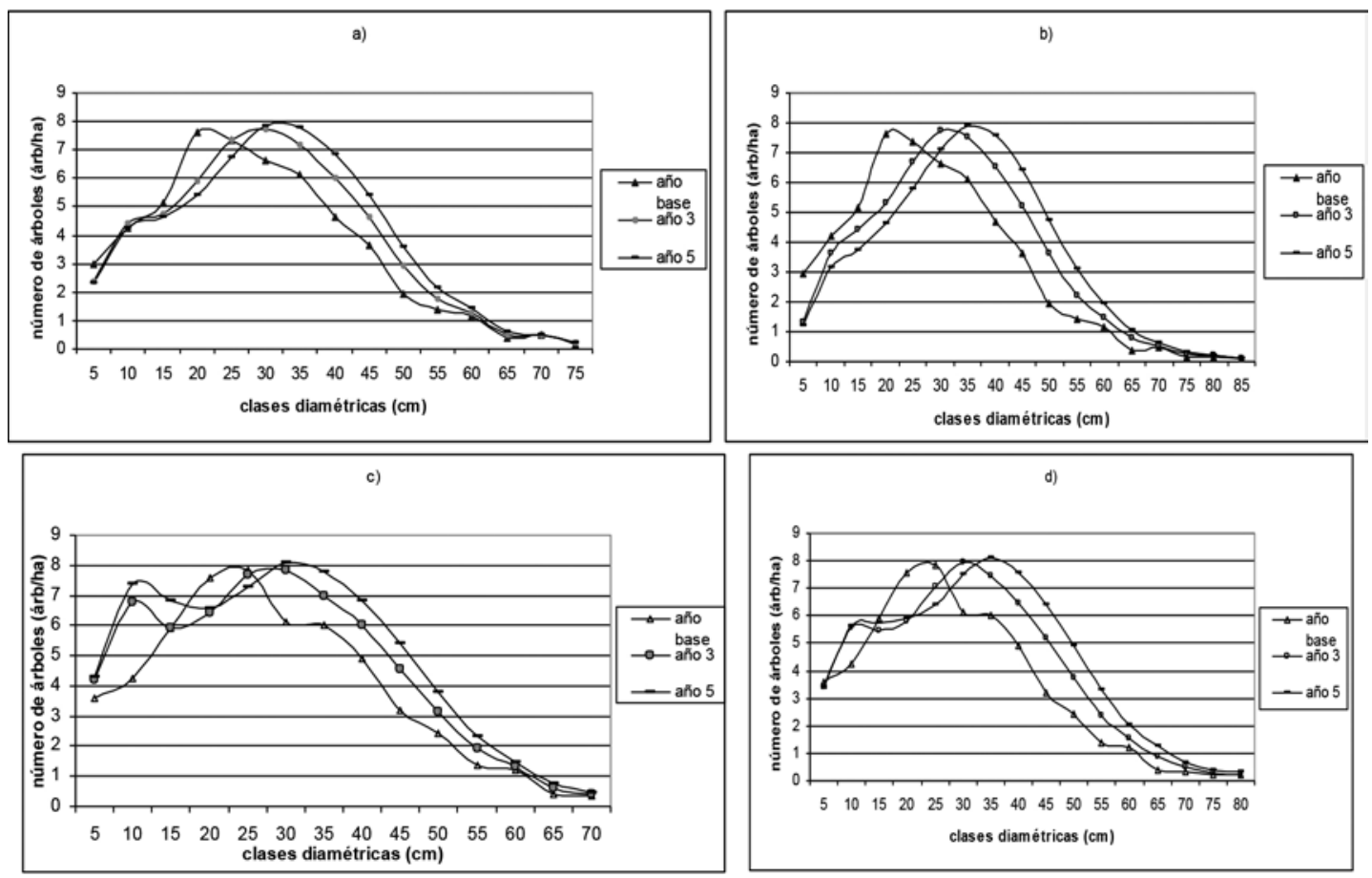

Figura 2. Proyección de la población de laurel para cinco años en a) Cacao ladera, b) Cacao valle, c) Banano ladera y d) Banano valle en Talamanca, Costa Rica.

La proyección del rendimiento de madera en volumen comercial a nivel de poblaciones fue creciente en todos los sistemas agroforestales, los mayores rendimientos se producirán en los sistemas agroforestales en valle, a cinco años de proyección los SAF en laderas estarán produciendo un 30\% de madera menos en relación a los SAF en valle (Cuadro 3).

Cuadro 3. Proyección de las existencias $\left(m^{3}\right.$ ha-1) de madera comercial de laurel.

\begin{tabular}{|l|l|l|l|l|l|l|}
\hline \multicolumn{1}{|c|}{ SAF } & \multicolumn{1}{c|}{ Año base } & \multicolumn{1}{c|}{ Año 1 } & \multicolumn{1}{c|}{ Año 2 } & \multicolumn{1}{c|}{ Año 3 } & \multicolumn{1}{c|}{ Año 4 } & \multicolumn{1}{c|}{ Año 5 } \\
\hline & $\left(\mathrm{m}^{3}\right.$ ha- 1$)$ & $\left(\mathrm{m}^{3}\right.$ ha- 1$)$ & $\left(\mathrm{m}^{3}\right.$ ha- 1$)$ & $\left(\mathrm{m}^{3}\right.$ ha- 1$)$ & $\left(\mathrm{m}^{3}\right.$ ha- 1$)$ & $\left(\mathrm{m}^{3}\right.$ ha- 1$)$ \\
\hline $\mathrm{CL}$ & 30,40 & 33,80 & 35,09 & 37,96 & 41,02 & 44,31 \\
\hline $\mathrm{CV}$ & 33,11 & 34,89 & 40,15 & 46,07 & 52,64 & 59,85 \\
\hline BL & 28,70 & 31,34 & 34,39 & 37,59 & 41,14 & 44,86 \\
\hline BV & 32,80 & 35,52 & 41,50 & 48,15 & 55,50 & 63,65 \\
\hline
\end{tabular}

CV: cacao en valle; CL: cacao en ladera; BV: banano valle; BL; banano ladera

El ICAVc fue creciente en todos los sistemas agroforestales a excepción del cacao en ladera que disminuyó en el tiempo, los BV fueron los que presentaron los mayores ICAVc. El incremento periódico anual del volumen comercial (IPAvc) será mayor en los sistemas agroforestales en valle (Cuadro 4). A nivel de 
población en cacaotales y bananales el aprovechamiento es sostenible puesto que hay incrementos en la producción de madera.

Cuadro 4. Proyección de los incrementos volumétricos de madera comercial (dap $\geq 45 \mathrm{~cm}$ ) de laurel.

\begin{tabular}{|l|l|l|l|l|l|l|}
\hline \multicolumn{1}{|c|}{ SAF } & \multicolumn{1}{|c|}{ ICAVc año1 } & \multicolumn{1}{c|}{ ICAVc año 2 } & \multicolumn{1}{c|}{ ICAVC año 3 } & \multicolumn{1}{c|}{ ICAVC año 4 } & \multicolumn{1}{c|}{ ICAVc año 5 } & \multicolumn{1}{c|}{ IPAvc } \\
\hline & $\left(\mathrm{m}^{3}\right.$ ha-1 $)$ & $\left(\mathrm{m}^{3}\right.$ ha-1 $)$ & $\left(\mathrm{m}^{3}\right.$ ha-1 $)$ & $\left(\mathrm{m}^{3}\right.$ ha- 1$)$ & $\left(\mathrm{m}^{3}\right.$ ha-1 $)$ & $\left(\mathrm{m}^{3}\right.$ ha-1 $)$ \\
\hline $\mathrm{CL}$ & 3,40 & 1,29 & 2,87 & 3,06 & 3,29 & 2,78 \\
\hline CV & 1,78 & 5,26 & 5,92 & 6,58 & 7,21 & 5,38 \\
\hline BL & 2,64 & 3,05 & 3,20 & 3,55 & 3,72 & 3,23 \\
\hline BV & 3,14 & 5,98 & 6,65 & 7,35 & 8,15 & 6,25 \\
\hline
\end{tabular}

CV: cacao en valle; CL: cacao en ladera; BV: banano valle; BL: banano ladera

La densidad de laurel disminuirá en el 40 y $50 \%$ de las parcelas de banano y cacao respectivamente. Mientras que el 14 y el 17\% de las parcelas de banano y cacao respectivamente tendrán altos incrementos de densidad; este resultado sugiere que el incremento de la densidad que se observa a nivel de poblaciones de SAF es producto del alto incremento de pocas parcelas en los sistemas agroforestales evaluados, mientras que en la mayoría de las parcelas la densidad está disminuyendo o tienen incrementos bajos (Figura 3).

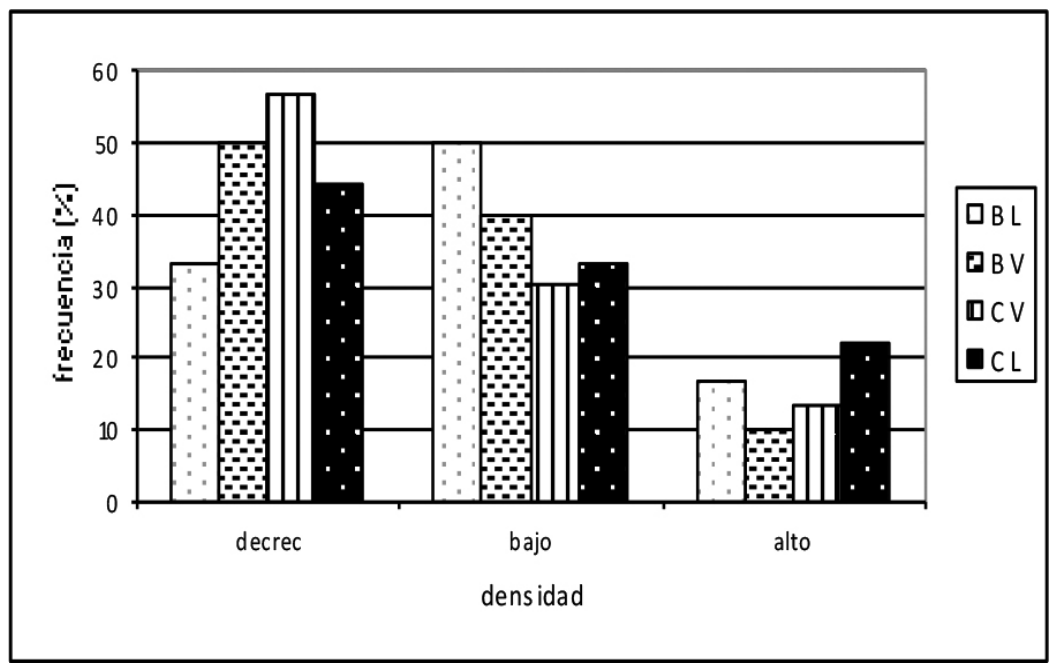

Figura 3. Comportamiento de la densidad de laurel a nivel de parcelas con una proyección a cinco años.

\section{Análisis de escenarios}

\section{Sin aprovechamiento de individuos $\leq 45 \mathrm{~cm}$ de dap}

En este escenario, considerando la sobrevivencia del 100\% de los individuos con dap $\leq 45 \mathrm{~cm}$ (Cuadro 5) se obtiene que en todos los sistemas agroforestales habrán incrementos en el ICAVc y el IPAvc, siendo los bananales los de mayores incrementos (13\% más que los cacaotales), sin embargo, los incrementos son bajos con respecto a la proyección con los datos observados. 
Cuadro 5. Proyección de los incrementos volumétricos IPAvc de madera comercial de laurel por 5 años

\begin{tabular}{|c|c|c|c|c|c|c|}
\hline SAF & $\begin{array}{l}\text { Año } 1 \\
\text { ICAVc }\end{array}$ & $\begin{array}{l}\text { Año } 2 \\
\text { ICAVC }\end{array}$ & $\begin{array}{l}\text { Año } 3 \\
\text { ICAVc }\end{array}$ & $\begin{array}{l}\text { Año } 4 \\
\text { ICAVC }\end{array}$ & $\begin{array}{l}\text { Año } 5 \\
\text { ICAVC }\end{array}$ & IPAvc \\
\hline & (m³a-1) & (m³a-1) & (m³ ha-1) & (m³a-1) & (m ha-1) & (m ha-1) \\
\hline $\mathrm{CL}$ & 2,17 & 2,63 & 2,95 & 3,87 & 2,84 & 2,99 \\
\hline CV & 4,53 & 5,35 & 6,18 & 6.82 & 7,59 & 6,09 \\
\hline$B L$ & 2,65 & 3,13 & 3,27 & 3,67 & 3,88 & 3,32 \\
\hline BV & 5,14 & 6,09 & 6,62 & 7,51 & 8,53 & 6,82 \\
\hline
\end{tabular}

CV: cacao en valle; CL: cacao en ladera; BV: banano valle; BL; banano ladera

\section{Incrementos del 50\% de reclutamiento de individuos}

En este escenario, se obtiene un incremento en el Vc de madera en todos los SAF evaluados siendo los bananales los que mayores incrementos presentan, no obstante, los sistemas en laderas no presentan incrementos cuando se compararan con las proyecciones de los datos observados (Cuadro 6).

Cuadro 6. Proyección de los incrementos volumétricos IPAvc de madera comercial de laurel por cinco años en Talamanca, Costa Rica.

\begin{tabular}{|c|c|c|c|c|c|c|}
\hline SAF & $\begin{array}{l}\text { Año } 1 \\
\text { ICAVC }\end{array}$ & $\begin{array}{l}\text { Año } 2 \\
\text { ICAVC }\end{array}$ & $\begin{array}{l}\text { Año } 3 \\
\text { ICAVC }\end{array}$ & $\begin{array}{l}\text { Año } 4 \\
\text { ICAVC }\end{array}$ & $\begin{array}{l}\text { Año } 5 \\
\text { ICAVC }\end{array}$ & IPAvc \\
\hline & (m³ ha-1) & (m³ ha-1) & (m³ ha-1) & (m³ ha-1) & (m³ ha-1) & ( $\left.m^{3} h a-1\right)$ \\
\hline $\mathrm{CL}$ & 3,30 & 1,20 & 2,70 & 3,00 & 2,99 & 2,67 \\
\hline CV & 4,53 & 5,35 & 6,18 & 6,82 & 6,09 & 6,09 \\
\hline$B L$ & 2,60 & 3,06 & 3,20 & 3,55 & 3,32 & 3,23 \\
\hline BV & 5,10 & 5,98 & 6,66 & 7,35 & 6,82 & 6,65 \\
\hline
\end{tabular}

CV: cacao en valle; CL: cacao en ladera; BV: banano valle; BL; banano ladera

\section{Proyección del rendimiento e incremento de madera y aplicación del modelo}

Los incrementos de madera tanto en cacaotales como en bananales son inferiores $(2,8 ; 5,4 ; 3,2$ y 6,25 $\mathrm{m}^{3}$ ha $^{-1}$ año-1 para CL, CV, BL y BV respectivamente) a los reportados en otros estudios realizados con laurel. Rosero y Gewald (1979) reportan incrementos en volumen de 14,8 m³ ha-1 año-1; Somarriba (1990) reporta incrementos en volumen de $9-24 \mathrm{~m}^{3}$ ha-1 año-1. Sin embargo, los resultados obtenidos en este estudio son superiores a la proyección realizada por Suárez (2001) para estas mismas parcelas.

La distribución de la población de laurel con datos observados muestra que la clase diamétrica de 20-25 $\mathrm{cm}$ tiene la mayor densidad de individuos y luego desciende hacia los diámetros superiores, siendo esto una característica de las poblaciones de regeneración natural.

La poca presencia de individuos en las clases diamétricas inferiores a $20 \mathrm{~cm}$ (figura 2), puede deberse a factores ecológicos y de manejo, los factores ecológicos estarían asociados a: la biomasa en descomposición sobre el suelo la cual incide en la viabilidad de la semilla; la sombra del dosel de árboles y del cacao y el periodo de lluvias puesto que el laurel germina en época seca y estas arrastraran muchas semillas. Los factores de manejo están asociados a: las frecuencia de las chapias que realiza el agricultor en la cual son eliminadas muchas plantitas; al raleo para regular sombra para el cacao o banano y que implica eliminar árboles pequeños, a partir de dap > $12 \mathrm{~cm}$ este raleo disminuye. En la proyección de la población de laurel para cinco años se observa la misma tendencia (Figura 2) e incluso la curva hace moda en $25 \mathrm{~cm}$.

En el análisis de los dos escenarios no se observan incrementos importantes en la producción de madera con respecto a los datos observados. Es probable que el periodo de análisis de cinco años es muy 
poco tiempo para que se exprese en el volumen de madera de laurel el efecto de mayores reclutamientos o el no aprovechamiento de individuos menores de $45 \mathrm{~cm}$ de dap. Con estas proyecciones, el sistema es sostenible porque hay incrementos de madera.

El modelo desarrollado puede considerarse como un reajuste al modelo de Suárez (2001), este consideró el reclutamiento como una nueva variable, calculó la sobrevivencia a partir de dos momentos de evaluación e incorporó datos de individuos desde las primeras clases diamétricas, por lo que el modelo gana en eficiencia.

\section{Conclusiones}

- El aprovechamiento de madera en estos sistemas agroforestales es sostenible, puesto que ocurren incrementos tanto en volumen de madera como en la densidad poblacional.

- Los sistemas agroforestales de cacao y banano orgánico de indígenas de Talamanca son fuentes importantes de madera.

- En el periodo proyectado con las matrices de transición, con las actuales tasas de extracción y reclutamiento, las poblaciones de laurel en los SAF aumentarán en (volumen y densidad).

- En los escenarios evaluados (disminución de la mortalidad e incrementos de las tasas de reclutamiento) los incrementos serán crecientes en todos los SAF.

- Los sistemas agroforestales evaluados son sostenibles con las actuales tasas de aprovechamiento y manejo.

- El modelo aplicado es una herramienta útil que permite evaluar la sostenibilidad de los sistemas productivos diversificados como son los SAF.

\section{Lista de referencias}

Albertin, A; Nair, PK. (2004). Farmers Perspectives on the role of shade Trees in Coffee Productions Systems: An assessment from the Nicoya Peninsula, Costa Rica. Human Ecology 32(4): 443-463.

Barrance, AJ; Flores, L; Padilla, E; Gordon, JE; Schreckenberg, K. (2003). Trees and farming in the dry zone of southern Honduras I: campesino tree husbandry practices. Agroforestry Systems 59: 97-106.

Camargo, JC; Ibrahim, M; Somarriba, E; Finegan, B; Current, D. (2000). Factores ecológicos y socioeconómicos que influyen en la regeneración natural del laurel en sistemas silvopastoriles del trópico húmedo y subhúmedo de Costa Rica. Agroforestería en las Américas 7(26):46-49.

Moscovich, FA. (2004). Modelos de crecimiento y producción forestal. INTA. Centro Regional Misiones, Estación Experimental Montecarlo, Argentina. 42p.

Nair, PK. (1997). Agroforestería. Centro de Agroforestería para el desarrollo sostenible. Universidad Autónoma de Chapingo, México. Editor L. Krishnamurthy. 543 p.

Pérez, CF. (1954). Estudio forestal del Laurel Cordia alliodora (Ruiz y Pavón) Cham., en Costa Rica. Tesis Mag. Sc. Turrialba, Costa Rica, IICA. 195 p.

Rosero, P; Gewald, N. (1979). Growth of laurel (Cordia alliodora) in coffe and cacao plantations, and pastures, in the Atlantic region of Costa Rica. In De las Salas G ed. Proceeding of the Workshop Agro-forestry Systems in Latin America. Turrialba, Costa Rica CATIE. pp 205-208.

Russell, D; Franzel, S. (2004). Trees of prosperity: Agroforestry, markets and the African smallholder. Agroforestry Systems 61: 345-355. 
Somarriba, E. (2001). Acacia pennatula en los potreros de la reserva natural Mesas de Moropotente, Estelí, Nicaragua. 28 p.

Somarriba, E. (1990). Sustainable timber production from uneven-aged shade stands of Cordia alliodora in small coffe farms. Agroforestry Systems 10: 253-263.

Somarriba, E; Beer, J. (1987). Dimensions, volumes and growth of Cordia alliodora in agroforestry systems. Forest Ecology and Management 18(2):113-126.
Suárez, A. (2001). Aprovechamiento sostenible de madera de Cordia alliodora y Cedrela odorata de regeneración natural en cacaotales y bananales de indígenas de Talamanca, Costa Rica. Tesis Mag. Sc. Turrialba, Costa Rica, CATIE. 77 p.

Suatunce, P. (2002). Diversidad de escarabajos estercoleros en bosques y en cacaotales de diferentes estructuras y composición florística, Talamanca, Costa Rica. Tesis Mag. Sc. Turrialba, Costa Rica, CATIE. 135 p.

Vanklay, J. (1994). Modelling forest growth and yield Applications to Mixed Tropical Forests. Cab International, Oxford, UK. 312 p. 